\title{
Mechanistic Study of the Hydrothermal Reduction of Palladium on the Tobacco Mosaic Virus
}

\author{
Oluwamayowa O. Adigun ${ }^{a}$, Alexander S. Freer ${ }^{a}$, Jeffrey T. Miller ${ }^{a}$, L. Sue Loesch-Fries ${ }^{b}$, \\ Bong Suk Kim ${ }^{b}$, Michael T. Harris ${ }^{\mathrm{a}, *}$ \\ ${ }^{a}$ School of Chemical Engineering, Purdue University, 480 Stadium Drive, Forney Hall, IN 47907 \\ ${ }^{b}$ Department of Botany and Plant Pathology, Purdue University, West Lafayette, IN, 47907
}

\begin{abstract}
The fundamental mechanisms governing reduction and growth of palladium on the genetically engineered Tobacco mosaic virus in the absence of an external reducer have been elucidated via in situ X-ray absorption spectroscopy. In recent years, many virusinorganic materials have been synthesized as a means to produce high quality nanomaterials. However, the underlying mechanisms involved in virus coating have not been sufficiently studied to allow for directed synthesis. We combined XAS, via XANES and EXAFS analysis, with TEM to confirm an autocatalytic reduction mechanism mediated by the TMV1Cys surface. This reduction interestingly proceeds via two first order regimes which result in two linear growth regimes as spherical palladium nanoparticles are formed. By combining this result with particle growth data, it was discovered that the first regime describes growth of palladium nanoparticles on the virion while the second regime describes a second layer of larger particles which grew sporadically on the first palladium nanoparticle layer. Subsequent aggregation of free solution based spherical particles and metallized nanorods characterize a third and final regime. At the end of the second reduction regime, the average particle diameter of particles tethered to the TMV1Cys surface are approximately $4.5 \mathrm{~nm}$. The use of XAS to simultaneously monitor the kinetics of biotemplated reactions along with growth of metal nanoparticles will provide insight into the pertinent reduction and growth mechanisms so that nanorod properties can be controlled through their populating nanoparticles.
\end{abstract}

(C) 2015 Published by Elsevier Ltd.

Keywords: X-ray Absorption Spectroscopy(XAS), in situ, mineralization, hydrothermal synthesis, Tobacco Mosaic Virus, reduction

*Corresponding author. Phone:+1 765494 0963, Fax: +1 7654940805.

Email addresses: oadigun@purdue.edu (Oluwamayowa O. Adigun), afreer@purdue.edu (Alexander S. Freer), jeffrey-t-miller@purdue.edu (Jeffrey T. Miller), loeschfr@purdue .edu (L. Sue Loesch-Fries), kim1@purdue.edu (Bong Suk Kim), mtharris@purdue.edu (Michael T. Harris)

${ }^{1}$ School of Chemical Engineering, Purdue University, West Lafayette

${ }^{2}$ Department of Botany and Plant Pathology, Purdue University, West Lafayette, Indiana, 47907 


\section{Introduction}

The use of biological templates as a means to produce organic-inorganic materials has become a prominent area of research in recent years. Nanomaterials engineered from DNA[1-5], virus[6$10]$ and protein fibril[11] templates have produced promising materials. In comparison with traditional methods for the production of nanorods, such as vapor-liquid-solid processing[12], chemical vapor deposition[13,14] and liquid-phase colloidal synthesis[15-17] that require extreme processing conditions (high temperature or vacuum conditions), biotemplating offers a means of production of materials under mild conditions.

Additionally, the easy availability and precise size of many biological templates combined with their malleability via bioengineering methods makes them potentially economically viable in the industry. Particularly, using biological nanostructures such as the Tobacco mosaic virus (TMV) as templates has attracted a lot of attention due to their applicability in the production of nanorods and nanowires. As a result, materials created from TMV have been applied to electroless deposition[18], battery electrodes[18, 19], memory devices[20], chemical sensing[21], catalysis[22] and sol-gel chemistry[23]. The TMV virion has proven to be a very simple and multi-functional biotemplate. It is known to consist of a protein nanotube surrounding an embedded RNA molecule[24]. The nanotube is $300 \mathrm{~nm}$ in length and $18 \mathrm{~nm}$ in diameter (with a $4 \mathrm{~nm}$ inner channel) and is developed from a helically assembled array of 2130 identical coat proteins (CPs). The interior of the tube contains an RNA molecule, which spans the length of the virus. On the surface of these viruses, the coat proteins, which are biopolymers of 158 amino acids, interact through mostly hydrogen bonds to assemble into a rod shape thereby exposing specific amino acids on the surface of the particle. The outer surface of the virus can be described as a simple repetition of identical $2.3 \times 3.5 \mathrm{~nm}^{2}$ unit surfaces[25]. The virion $\mathrm{CP}$ has been engineered via site directed mutagenesis[26] so that one or two cysteine groups can be exposed on the outer surface of each coat protein. The subsequent coating of these genetically modified TMVs (TMV1Cys and TMV2Cys) as well as wild type TMV with various inorganic nanoparticles has been widely reported[18, 23, 27-35].

As TMV and its mutants are being applied as biotemplates, the importance of understanding 
the nanoparticle coating process is becoming increasingly clear. Platinum nanocluster deposition on TMV showed memory storage properties believed to be caused by the nanoparticle sizedependent coulomb blockage effect with the RNA aromatic groups [20]. TMV virions which were assembled on gold substrates were coated with palladium nanoparticles followed by an electroless deposition of nickel before being applied as a battery electrode[18]. More recently, TMV virions conjugated with different sizes of palladium[36] and gold[28] nanoparticles have been electrically characterized in view of applying them to nanocircuits. Furthermore, these same nanomaterials have been shown to be extremely attractive in metal surface-mediated catalysis due to both (1) the ideal(approximately 2 - 5nm) sizes of tethered populating particles for high catalytic activity and (2) the ability to display such particles at high surface areas per mass of precious metal without their aggregation.[37, 38]. All of the studies utilizing these particular applications imply that the pertinent properties of the biotemplated nanomaterials heavily depend on the size and surface coverage of nanoparticles on TMV.

The processes governing both of these are the adsorption, reduction and crystal growth mechanisms on the CPs of the biotemplate. However, the quantitative study on sorption of Pd (II) and $\mathrm{Au}$ (IV) by Lim et al.[33] and the X-ray scattering studies by Yi and co-workers[34? ] are the only works germane to any of these mechanisms. Also, research has not been conducted to study the relationship between these mechanisms even though it is widely agreed that all occur at some point in the mineralization process. Instead, many findings have been explained by conjecture which may not fully describe the entire process due to the complexity of the protein surfaces at work.

For example, although biosorption on TMV is generally postulated to occur by anionic metal deposition on TMV via simple electrostatic attraction by positively charged amino acid groups[30, 39], studies on the engineered virus have shown that the thiol functionality from cysteine groups increased the decorated gold, silver and palladium clusters compared to wild type TMV[32, 33]. Parallel studies performed on mineralization of Pd on functionalized silica further confirm that thiol groups are more involved in the mineralization process than the electrostatic model had initially assumed[40]. Similar results have been observed from sorption of gold by polyhistidine-modified TMV-like nanorods[28]. In general, other groups on virus surfaces are reported to show affin- 
ity for many inorganic materials. Individual amino acids or peptides containing tyrosine[35, 41], tryptophan, serine[42], aspartic acid and glutamic acid[43] have been implicated in these systems. This is further evidenced in the biosorption experiments perfomed by Peelle et al.[6] Additionally, the ability for nearby amino acids to upregulate and downregulate biosorption is a phenomenon that has not been accounted for in current biotemplating models.

Lim et al.[44] made a case for the study of reduction on the TMV surface when they observed pronounced $\mathrm{Pd}$ reduction in the absence of an external reducing agent (we are now referring to this synthesis as the hydrothermal method). The nanorods synthesized, which had more surface coverage, greater uniformity and smaller nanoparticles than any previously synthesized, are ideal for many applications. However, the reduction mechanism (which may be coupled with biosorption) that led to these high quality rods is still unknown. The work thus proves the attainability of very high quality nanorods and encourages studies that produce experimental data to explain how reduction and particle growth occur simultaneously. Following this study, TEM studies performed by other researchers[45] showed that the formation of Pd on the surface of TMV1Cys in the absence of a reducing agent can be difficult to predict. Along with these work, other sources[35, 46-48] have also reported reduction and particle formation of metal precursors on biological templates in the absence of an external reducing agent. Therefore, clarification of this yet unexplained but prevalent phenomenon in the metallization of biotemplates is needed.

X-ray absorption spectroscopy (XAS) is a technique that has successfully been used to study nanoparticle formation mechanisms[49-52]. Despite its potential, its use has not yet been explored in the field of biotemplating. This is because the low concentrations of inorganic materials used in biotemplating are only recently detectable in the most advanced of synchrotron X-ray research facilities. Also, most biotemplating reactions occur rapidly due to the ubiquitous use of reducing agents, so that they leave very little room for observation of dynamic reaction activity. For systems at suitably high concentrations and slower reaction rates, XAS can provide information on coordination number, concentration of species and oxidation states during the course of a reaction. These will provide insight into the mechanism of growth, kinetics of reduction and possibly even intermediate reduction states during reaction. In this study, the hydrothermal synthesis which we previously discovered[44] is monitored using XAS. X-ray absorption spectra are collected over 
time and are then analyzed to study the extended X-ray absorption fine structure (EXAFS) and Xray absorption near edge spectroscopy (XANES) regions. These are used to characterize reduction and growth throughout the coating process.

\section{Materials and Methods}

TMV1Cys was purified with a few modifications from the protocol described by Yi et al.[26] A $1.5 \mathrm{~mL}$ solution containing $3.5 \times 10^{-1} \mathrm{mg} / \mathrm{mL}$ of TMV1Cys and $5 \mathrm{mM}$ sodium tetrachloropalladate (Na2PdC14) (purchased from Sigma Aldrich) aqueous solution was placed in a $2 \mathrm{~mL}$ reaction vessel. The mixture was continuously stirred. The palladium precursor concentration was increased significantly compared to previous studies $[44,53]$ so that it surpassed the detectable limit of X-ray detectors. Also, the $[\mathrm{TMV} 1 \mathrm{Cys}]$ to $[\mathrm{Pd}] \operatorname{ratio}([\mathrm{g} / \mathrm{L}]:[\mathrm{mol} / \mathrm{L}])$ was doubled. The vessel was then placed in a cell at $57^{\circ} \mathrm{C}$ in the path of X-rays.

XAS measurements of the vessel and reference materials used were taken in transmission mode near the Pd K-edge (24.4 keV) with quick scans every 4 minutes. 178 absorption spectra were collected over a period of 18 hours during which a clear change in spectra is noticed as the species in solution evolve over time. Each spectrum was then analyzed individually but identically. The Pd K-edge energy was determined from the maximum of the first derivative in the XANES region after the normalized energy spectra was obtained. The EXAFS region and the XANES spectra were analyzed by standard procedures using WINXAS 3.1 fitting software. A palladium foil and palladium(II)chloride were used as references to obtain experimental phase shift and backscatter-

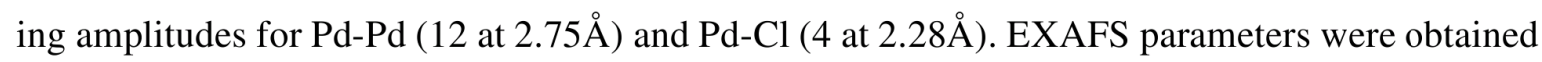
by performing a least square fit of Fourier transformed $\mathrm{k}^{2}$-weighted normalized absorption data in R-space. XANES data was used to obtain the ratio of oxidation states during the course of the reaction and EXAFS gave insight into species of Pd existing in solution and their average coordination number.

The energy of the XANES region used during analysis was from $24.31-24.39 \mathrm{keV}$. These were compared with the precursor solution $\left(\mathrm{Pd}^{2+}\right)$ and the final spectrum $\left(\mathrm{Pd}^{(0)}\right)$ after it was confirmed to be $100 \%$ Pd using a Pd foil. Each spectrum was then fit by assuming a linear combination of the two reference spectra. Pd-Cl and Pd-Pd bond phase and amplitude were used to fit each EXAFS 
spectra for the first 6 hours of data collection. After this point, Pd-Pd species properties were analyzed alone since XANES analysis showed that the concentration of $\mathrm{Pd}-\mathrm{Cl}$ species was below the detectable limit. Also, the spectra were continuously compared to $\mathrm{PdO}$ reference spectra to identify the presence of $\mathrm{Pd}-\mathrm{O}$ and $\mathrm{Pd}-\mathrm{Pd}(\mathrm{O})$ species during the reaction. The nanorods were then washed by redispersing them in $15 \mathrm{~mL}$ of water five times before transmission electron microscope (TEM) images were taken using a $200 \mathrm{kV}$ Tecnai T20 transmission electron microscope. Samples were prepared by depositing a 3-4 $\mu \mathrm{L}$ drop of TMV-Pd solution onto carbon formvar copper grids. Unwashed samples were examined to observe the particles in solution along with the nanorods.

\section{Results and discussion}

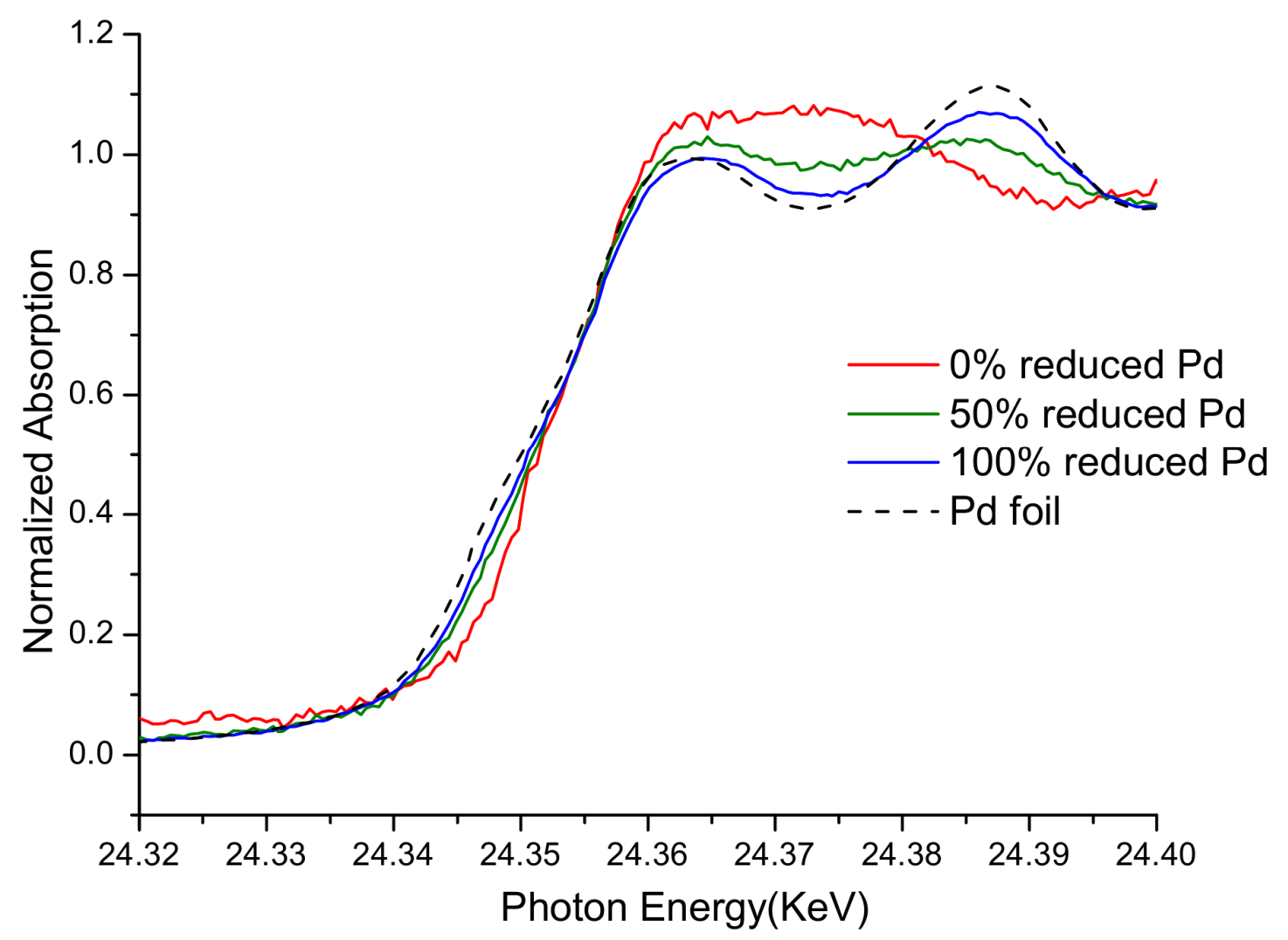

Figure 1. XANES Absorption Spectras showing evolution of Pd during TMV1Cys-mediated reduction. 


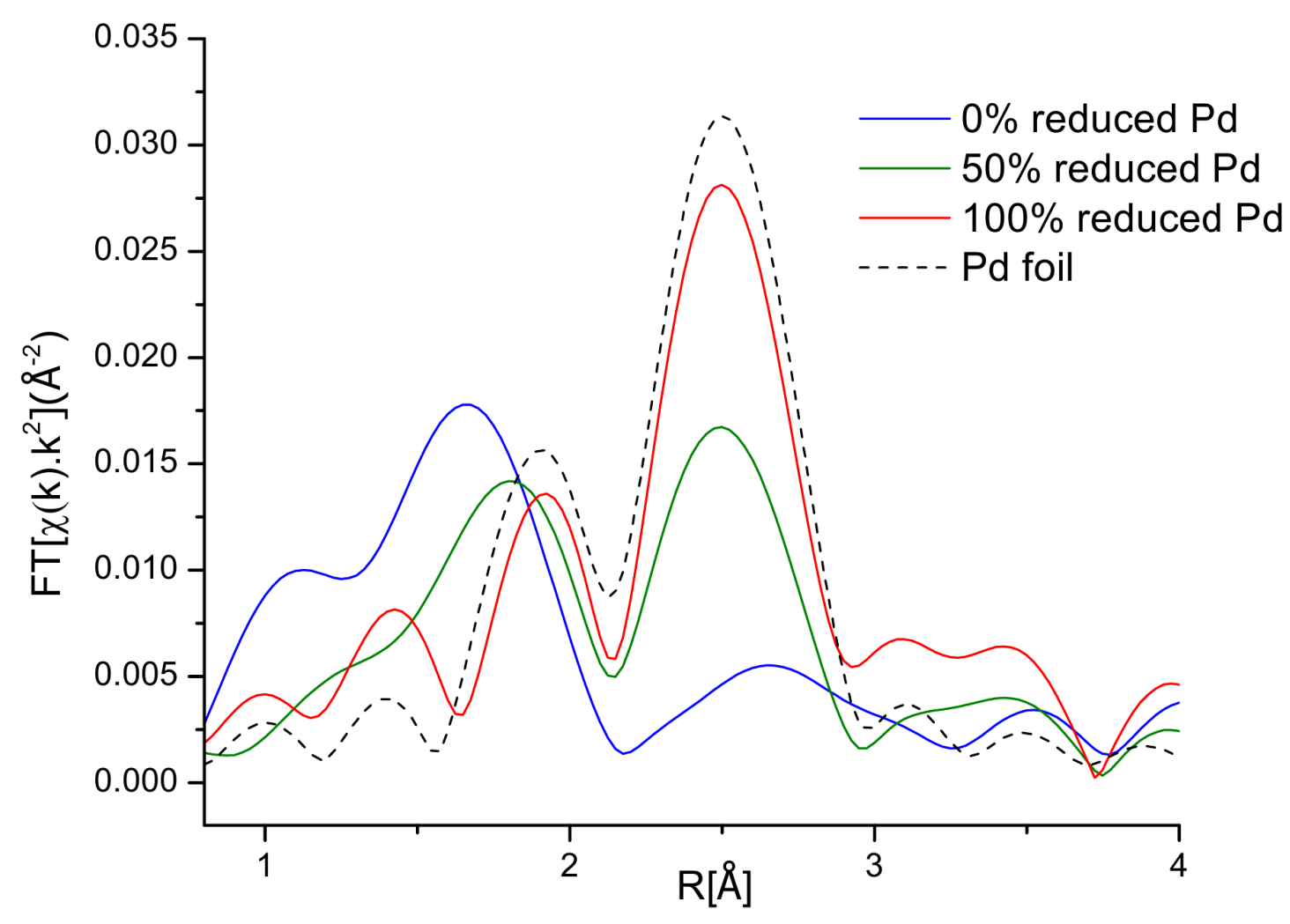

Figure 2. EXAFS spectras showing observed reduction of palladium.

The XANES and EXAFS spectras are shown for three time points during the reduction process in Figure 1 and Figure 2 respectively. A clear change in spectra from the palladium chloride species to reduced palladium is evident from the shapes of the curves. From the XANES analysis, no reaction is observed to occur within the first 1.8 hours. The existence of this induction period indicates that the reduction is accelerated by the presence of products i.e. the reaction is autocatalytic. After this, a rapid increase in $\mathrm{Pd}^{(0)}$ concentration is observed. No intermediate oxidation states or species are seen during this time. The reduction proceeds for the next 5.5 hours as shown in Figure 3(a). The plot of $\log [\mathrm{Pd}] /[\mathrm{Pd}]_{0}$ reduction against time shows two distinguishable linear regions indicating that the reduction is divided into two first order regimes with different rate constants of $0.30 \pm 0.017 \mathrm{hr}^{-1}$ and $0.09 \pm 0.008 \mathrm{hr}^{-1}$ respectively. In the analysis in this paper, the systems final spectrum was compared to that of a palladium foil as is shown in figure 2 . The spectra are very identical and thus led to a confirmation of close $100 \%$ conversion at the end of 
the reaction. This information is particularly important because it confirms the unique possibility of loading the precious metal on TMV1Cys to produce high quality nanowires without wastage.

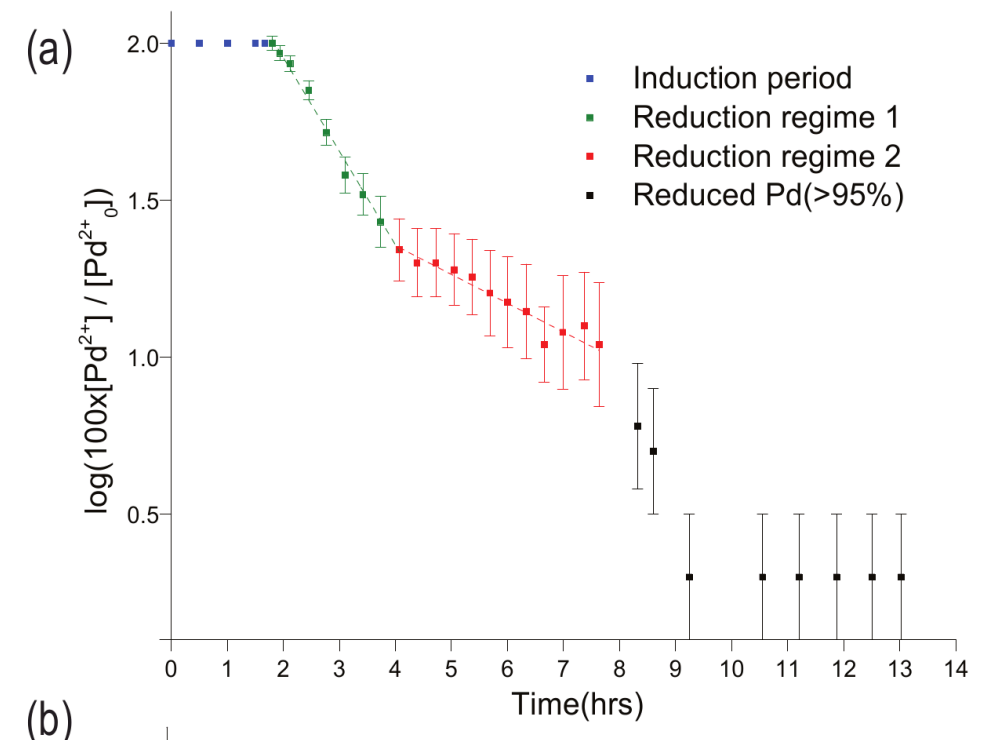

(b)

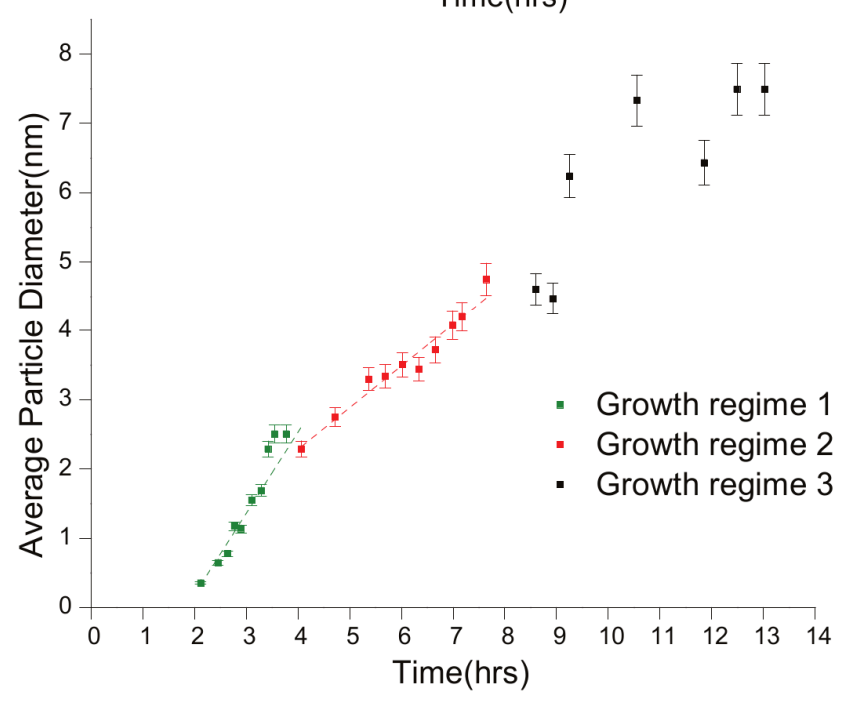

Figure 3. (a) Reduction of Palladium on TMV1Cys. The first order rate constants during reduction are $0.30 \pm 0.013 \mathrm{hr}^{-1}$ for regime 1 and $0.09 \pm 0.008 \mathrm{hr}^{-1}$ for regime 2 after which conversion of Pd is greater than $95 \%$. (b) Evolution of particle sizes in solution as reduction occurs. The linear growth rates are $1.2 \pm 0.08 \mathrm{nmhr}^{-1}$ for the first regime and $0.6 \pm 0.04 \mathrm{nmhr}^{-1}$ for the second regime. Palladium is reduced( $i 95 \%$ conversion) after the regime 2 .

From EXAFS analysis, the solution species fit to give reasonable bond lengths, coordination 
numbers and Debye Waller factors for both Pd-Pd and Pd-Cl. These properties are shown as they evolve during the course of reaction in Table 1(supporting information). The species evolve from initially $4 \mathrm{Pd}-\mathrm{Cl}$ bonds per $\mathrm{Pd}$ atom to a coordination number of 11.0 for $\mathrm{Pd}-\mathrm{Pd}$ at the end of the process. The presence of Pd-L (where $\mathrm{L}$ is any other atom in solution except $\mathrm{Cl}$ and $\mathrm{Pd}$ ) species was not observed suggesting that they could have been present only in low concentrations below the detectable limit of the technique. Particularly, the conspicuous absence of observable Pd-O (previously reported)[44] on the nanorods is useful information for the use of the nanorods in sensor applications. Also, a Pd-S species which has been observed via X-ray Photoelectron Spectroscopy (XPS)[44] was not distinguished because of the similarity in size between $\mathrm{Cl}$ and $\mathrm{S}$ atoms which would result in identical scattering of the photoelectron waves. Most of the nanorods are covered with approximately one layer of palladium nanoparticles across the TMV1Cys surface, as shown in transmission emission microscopy (TEM) image of produced nanorods in Figure 4. They can thus be approximated as a rod shape covered with a monolayer coating of spherical particles. This approximation allowed us to use the coordination numbers obtained from EXAFS to calculate the average particle size based on a model for free spherical particles developed by Miller et al.[52]. The model was obtained by correlating the dispersions obtained from hydrogen chemisorption with the coordination numbers obtained from XAS. The evolution of average particle size from the subsequent application of the model is shown in Figure 3(b).

Three linear regimes of particle growth can be distinguished during the course of growth (after the induction period). First, for the 1st 2 hours following the induction period, the growth of particles occurs at a rate of $1.2 \mathrm{~nm} / \mathrm{hr}$. The average palladium spherical nanoparticle diameter at the end of this period is about $2.5 \mathrm{~nm}$. The regime also correlates with the first regime in the reduction data (discussed above). The second regime of growth occurs for the next 3.5 hours. The growth rate during this time is $0.6 \mathrm{~nm} / \mathrm{hr}$. Like in the first regime, the growth in this time period also correlates with the second reduction regime. The significance of each of the regimes was surmised from a combination of TEM observations and the reduction and particle growth data.

The assembled TMV1Cys provides a surface for potential reduction. Along with it, there are also errant coat proteins and assembled TMV1Cys coat protein complexes (e.g. the 20S subunit) which could provide surfaces for nucleation. Since particle growth is not observed in the absence 

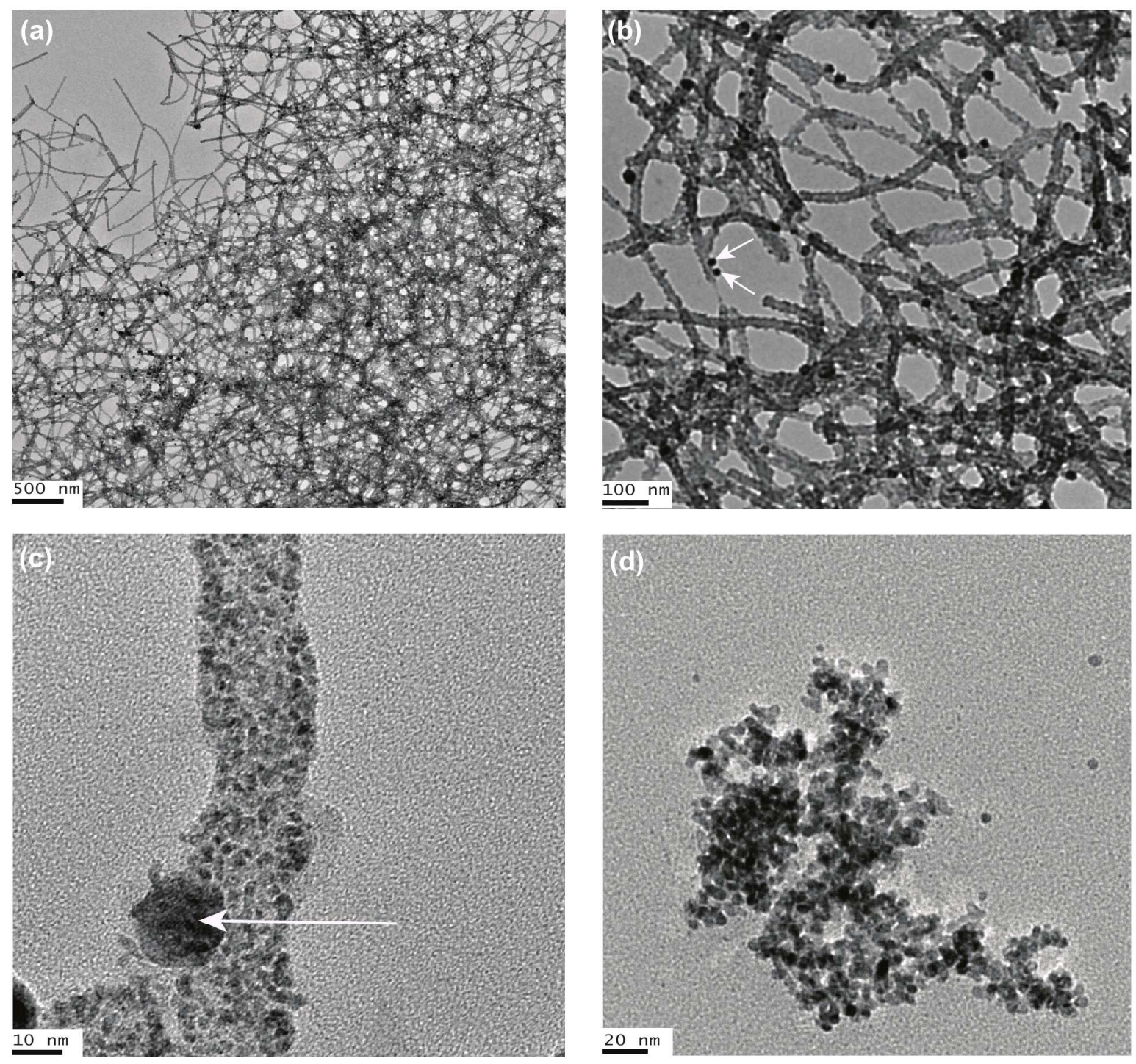

Figure 4. TEM images of particles in solution. All aliquots were taken after XAS experiment (at 14hr-timepoint). Arrows represent location of type II particles. (a) Low magnification image of washed TMV-Pd nanoparticles after the hydrothermal synthesis. (b) Washed TMV-Pd nanoparticles with clear observation of the type II particles grown on the initial layer of nanoparticles after the hydrothermal synthesis. (c) A single washed TMV1Cys-Pd nanorod showing first coating layer with particles of average size $2.5 \mathrm{~nm}$ along with larger type II particles. (d) Pd nanoparticles observed in solution when particles are not washed.

of the virus, interaction with these three surfaces must be responsible for the inception of both reduction and growth of palladium. Also the observation that the first regime has a higher reaction 
rate and faster growth than in the second regime indicates that two different mechanisms dominate during these two time periods. The average palladium spherical nanoparticle diameter after the first regime agrees with the average sizes of particles $(\approx 2.5 \mathrm{~nm})$ observed directly in contact with the TMV surface (Figure 4(c)). 2.5nm is the approximated projected length along the coat protein surface. Therefore, it is likely that the fast reduction/growth in the first regime results in the production of the $\approx 2.5 \mathrm{~nm}$ spherical particles observed. The change in particle growth to a slower growth (second regime) can be explained by a quenching of this growth/reduction behavior possibly after the growing particles collide as they grow on the TMV1Cys surface to which they are tethered. This theory is corroborated by both Lim et al.[44] and Freer et al.[53] who reported that the diameters of the nanorods on TMV2Cys-Pd stopped increasing significantly after the $\mathrm{pH}$ of the solution reached approximately 3.5 even in the presence of unreduced palladium precursor.

In the second regime, the continued growth and reduction rate can be explained by growth on top of the first layer Pd particles which grew on the TMV1Cys during the first regime. During this period, the reduction and growth data indicate that precursor is still being consumed to facilitate nanoparticle growth and it is also apparent that the particles directly in contact with the TMV surface and others found in solution (Figure 4(d)) are less than the $\approx 5 \mathrm{~nm}$ size found at the end of the growth regime. Close observation of TMV1Cys-Pd images of washed particles shows other larger 8-15nm spherical Pd particles (we will call these type II particles), as seen in Figures 4(b) and 4(c), which are attached to the smaller particles on the TMV1Cys surface. Deductions which we have made from the TEM images convince us that the growth of these particles is most likely described in the second regime. First, since they are all observed on top of the smaller particles they must have grown after them (i.e. in the second or third regime). Second, the sporadic growth all over the first Pd nanoparticle layer means that there are less of these particles and would imply a slower increase in average nanoparticle size and less consumption as observed in the second regime growth and reduction data. The existence of growth off the two different surfaces in the first and second regime explains why the usually linear region during autocatalytic growth[54] is instead characterized by two linear regions as shown in figure 3 .

The third regime in the growth data shows substantial variability. Such behavior is a result of small fluctuations in the EXAFS spectra from one time point to another. Physically, this would 


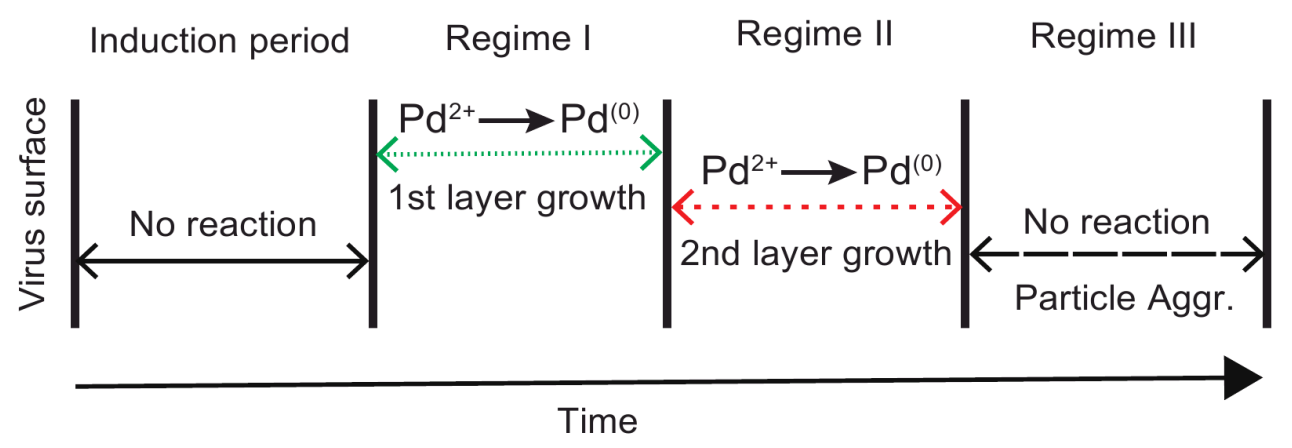

Figure 5. Proposed mechanism for TMV1Cys-mediated palladium reduction

have resulted from aggregation between particles in solution since all the Pd ions have already been reduced. Along with the TMV1Cys-Pd nanorods, free spherical particles which were not on the TMV1Cys surface were observed in the solution. These are shown in Figure 4(d). These free solution spherical particles could have nucleated on CPs or 20S CP complexes during the first regime. However, free from the constraints on the attached TMV1Cys particles which are tethered to a surface, these particles continue to aggregate into the third regime where their aggregating effect is observed. We surmise that haphazard aggregation of these particles as well as the coated nanorods is responsible for the continued changes in the EXAFS spectra. Aggregations of nanorods and nanoparticles in this way would weaken the assumption that the reduced Pd species can be approximated as spherical nanoparticles. Therefore, variability, as such, is expected at later times. Future work in the field would involve recognising this aggregation and innovating means to collect particles before its onset(e.g poly-L-lysine wrapping used by [35] on TMV-Au nanorods) so that individual nanorods can be collected for required applications.

\section{Conclusion}

In conclusion, this study has served as an entryway for detailed understanding of inorganic mineralization on biotemplates. Valuable information was gathered from the XAS study of the hydrothermal reduction of Pd on TMV1Cys. At the conditions studied, the reduction was first order and particle growth was linear. Additionally, because it allowed the simultaneous collection of re- 
duction and particle growth data, this study is the first study to observe an induction period before particles started to grow on any TMV virion. We can therefore confirm an autocatalytic reduction mechanism. The process involves two reduction regimes and three growth regimes which suggest different growth surfaces. By combining this information with TEM observations, we made deductions about the nature of particle growth on the virion surface These are summarized in figure 5. Further studies need to be conducted at different temperatures, concentrations (virus and metal precursor) and on more virions (wild type, TMV2Cys) to fully elucidate the hydrothermal synthesis by deciphering the reducing mechanism. This will allow the acquisition of fundamental knowledge for the control of particle sizes, surface coverage and uniformity on biotemplated metallized nanomaterials.

\section{Acknowledgements}

The authors thank Dr. James Culver at the University of Maryland Biotechnology Institute, Center for Biosystems Research for providing the TMV1Cys. This research used resources of the Advanced Photon Source, a U.S. Department of Energy (DOE) Office of Science User Facility operated for the DOE Office of Science by Argonne National Laboratory under Contract No. DE-AC02-06CH11357. XAS data was collected at the X-ray Operations and Research beamline 10-ID-B at the Advanced Photon Source, Argonne National Laboratory.

\section{References}

[1] T. Torimoto, M. Yamashita, S. Kuwabata, T. Sakata, H. Mori, H. Yoneyama, Fabrication of CdS Nanoparticle Chains along DNA Double Strands, The Journal of Physical Chemistry B 103 (42) (1999) 8799-8803. doi:10.1021/jp991781x.

URL http://pubs . acs . org/doi/abs/10.1021/jp991781x

[2] R. Mohammadzadegan, H. Mohabatkar, M. H. Sheikhi, A. Safavi, M. B. Khajouee, DNA-templated gold nanowires, Physica E: Low-dimensional Systems and Nanostructures 41 (1) (2008) 142-145. doi:10.1016/j.physe.2008.06.013.

URL http://linkinghub.elsevier.com/retrieve/pii/S1386947708002269

[3] J. Richter, M. Mertig, W. Pompe, H. Vinzelberg, Low-temperature resistance of DNA-templated nanowires 728 (2002) 725-728. 
[4] C. F. Monson, A. T. Woolley, DNA-Templated Construction of Copper Nanowires, Nano Letters 3 (3) (2003) 359-363. doi:10.1021/n1034016+.

URL http://pubs . acs .org/doi/abs/10.1021/n1034016\%2B

[5] S. Cui, Y. Liu, Z. Yang, X. Wei, Construction of silver nanowires on DNA template by an electrochemical technique, Materials \& Design 28 (2) (2007) 722-725. doi:10.1016/j.matdes.2005.08.007.

URL http://linkinghub.elsevier.com/retrieve/pii/S0261306905002323

[6] B. R. Peelle, E. M. Krauland, K. D. Wittrup, A. M. Belcher, Design criteria for engineering inorganic material-specific peptides., Langmuir : the ACS journal of surfaces and colloids 21 (15) (2005) 6929-33. doi:10.1021/la050261s.

URL http://www.ncbi.nlm.nih.gov/pubmed/16008405

[7] S. R. Whaley, D. S. English, E. L. Hu, P. F. Barbara, A. M. Belcher, Selection of peptides with semiconductor binding specificity for directed nanocrystal assembly, Nature 405 (2000) 665-668.

[8] A. B. Sanghvi, K. P. H. Miller, A. M. Belcher, C. E. Schmidt, Biomaterials functionalization using a novel peptide that selectively binds to a conducting polymer., Nature materials 4 (6) (2005) 496-502. doi:10.1038/nmat 1397.

URL http: //www.ncbi.nlm.nih.gov/pubmed/15895095

[9] C. E. Flynn, C. Mao, A. Hayhurst, J. L. Williams, G. Georgiou, B. Iverson, A. M. Belcher, Synthesis and organization of nanoscale II-VI semiconductor materials using evolved peptide specificity and viral capsid assemblyElectronic supplementary information (ESI) available: table of observed amino acid group frequency by position; Fig. 6. See ht, Journal of Materials Chemistry 13 (10) (2003) 2414. doi:10.1039/b307593a.

URL http://xlink.rsc.org/?DOI=b307593a

[10] C. Mao, D. J. Solis, B. D. Reiss, S. T. Kottmann, R. Y. Sweeney, A. Hayhurst, G. Georgiou, B. Iverson, A. M. Belcher, Virus-based toolkit for the directed synthesis of magnetic and semiconducting nanowires., Science (New York, N.Y.) 303 (5655) (2004) 213-7. doi:10.1126/science.1092740.

URL http://www.ncbi.nlm.nih.gov/pubmed/14716009

[11] J. Juárez, A. Cambón, A. Topete, P. Taboada, V. Mosquera, One-dimensional magnetic nanowires obtained by protein fibril biotemplating., Chemistry (Weinheim an der Bergstrasse, Germany) 17 (26) (2011) 7366-73. doi:10.1002/chem.201003679.

URL http://www.ncbi.nlm.nih.gov/pubmed/21557350

[12] J. Hu, T. W. Odom, C. M. Lieber, Chemistry and Physics in One Dimension: Synthesis and Properties of Nanowires and Nanotubes, Accounts of Chemical Research 32 (5) (1999) 435-445. doi:10.1021/ar9700365.

URL http://pubs . acs .org/doi/abs/10.1021/ar9700365

[13] X. Duan, C. M. Lieber, General Synthesis of Compound Semiconductor Nanowires, Advanced Materials 12 (4) (2000) 298-302. doi:10.1002/(SICI)1521-4095(200002)12:4¡298::AID-ADMA298;3.0.CO;2-Y. 
URL http://doi.wiley.com/10.1002/\%28SICI \%291521-4095\%28200002\%2912\%3A4\%3C298\%3A\%3AAID-ADMA298\%3E3

[14] J. Goldberger, R. He, Y. Zhang, S. Lee, Single-crystal gallium nitride nanotubes, Nature 422 (6932) (2003) 599-602. doi:10.1038/nature01484.1.

[15] P. Alivisatos, The use of nanocrystals in biological detection., Nature biotechnology 22 (1) (2004) 47-52. doi:10.1038/nbt927.

URL http://www.ncbi.nlm.nih.gov/pubmed/14704706

[16] W. U. Huynh, J. J. Dittmer, a. P. Alivisatos, Hybrid nanorod-polymer solar cells., Science (New York, N.Y.) 295 (5564) (2002) 2425-7. doi:10.1126/science.1069156.

URL http://www.ncbi.nlm.nih.gov/pubmed/11923531

[17] J. M. Caruge, J. E. Halpert, V. Wood, V. Bulović, M. G. Bawendi, Colloidal quantum-dot light-emitting diodes with metal-oxide charge transport layers, Nature Photonics 2 (4) (2008) 247-250. doi:10.1038/nphoton.2008.34. URL http://www . nature.com/doifinder/10.1038/nphoton.2008.34

[18] E. Royston, A. Ghosh, P. Kofinas, M. T. Harris, J. N. Culver, Self-assembly of virus-structured high surface area nanomaterials and their application as battery electrodes., Langmuir : the ACS journal of surfaces and colloids 24 (3) (2008) 906-12. doi:10.1021/la7016424.

URL http://www.ncbi.nlm.nih.gov/pubmed/18154364

[19] X. Chen, K. Gerasopoulos, J. Guo, A. Brown, C. Wang, R. Ghodssi, J. N. Culver, Virus-enabled silicon anode for lithium-ion batteries., ACS nano 4 (9) (2010) 5366-72. doi:10.1021/nn100963j.

URL http://www.ncbi.nlm.nih.gov/pubmed/20707328

[20] R. J. Tseng, C. Tsai, L. M, J. Ouyang, C. S. Ozkan, Y. Yang, Digital memory device based on tobacco mosaic virus conjugated with nanoparticles., Nature nanotechnology 1 (1) (2006) 72-7. doi:10.1038/nnano.2006.55.

URL http://www.ncbi.nlm.nih.gov/pubmed/18654145

[21] M. A. Bruckman, J. Liu, G. Koley, Y. Li, B. Benicewicz, Z. Niu, Q. Wang, Tobacco mosaic virus based thin film sensor for detection of volatile organic compounds, Journal of Materials Chemistry 20 (27) (2010) 5715. doi:10.1039/c0jm00634c.

URL http://xlink.rsc.org/?DOI=c0jm00634c

[22] C. Yang, A. K. Manocchi, B. Lee, H. Yi, Viral-templated palladium nanocatalysts for Suzuki coupling reaction, Journal of Materials Chemistry 21 (1) (2011) 187. doi:10.1039/c0jm03145c.

URL http: //xlink.rsc.org/?DOI=c0jm03145c

[23] E. S. Royston, A. D. Brown, M. T. Harris, J. N. Culver, Preparation of silica stabilized Tobacco mosaic virus templates for the production of metal and layered nanoparticles., Journal of colloid and interface science 332 (2) (2009) 402-7. doi:10.1016/j.jcis.2008.12.064.

URL http://www .ncbi.nlm.nih.gov/pubmed/19159894

[24] P. Butler, A. Klug, Assembly of the Particle of Tobacco Mosaic Virus from RNA and Disks of Protein, Nature 
New Biology 229 (2) (1971) 47-50. doi:doi:10.1038/newbio229047a0.

[25] K. Namba, R. Pattanayek, G. Stubbs, Visualization of protein-nucleic acid interactions in a virus. Refined structure of intact tobacco mosaic virus at 2.9 A resolution by X-ray fiber diffraction., Journal of molecular biology 208 (2) (1989) 307-25.

URL http://www.ncbi.nlm.nih.gov/pubmed/2769760

[26] H. Yi, S. Nisar, S.-Y. Lee, M. A. Powers, W. E. Bentley, G. F. Payne, R. Ghodssi, G. W. Rubloff, M. T. Harris, J. N. Culver, Patterned assembly of genetically modified viral nanotemplates via nucleic acid hybridization., Nano letters 5 (10) (2005) 1931-6. doi:10.1021/n1051254r.

URL http://www.ncbi.nlm.nih.gov/pubmed/16218712

[27] S. Y. Lee, J. Choi, E. Royston, D. B. Janes, J. N. Culver, M. T. Harris, Deposition of Platinum Clusters on Surface-Modified Tobacco Mosaic Virus, Journal of Nanoscience and Nanotechnology 6 (4) (2006) 974-981. doi:10.1166/jnn.2006.146.

URL http://www. ingentaselect.com/rpsv/cgi-bin/cgi?ini=xref\&body=linker\&reqdoi=10.1166/jnn .2006.14

[28] M. Wnek, M. L. Górzny, M. B. Ward, C. Wälti, A. G. Davies, R. Brydson, S. D. Evans, P. G. Stockley, Fabrication and characterization of gold nano-wires templated on virus-like arrays of tobacco mosaic virus coat proteins., Nanotechnology 24 (2) (2013) 025605. doi:10.1088/0957-4484/24/2/025605.

URL http://www.ncbi.nlm.nih.gov/pubmed/23220929

[29] W. Shenton, T. Douglas, M. Young, G. Stubbs, S. Mann, Inorganic-Organic Nanotube Composites from Template Mineralization of Tobacco Mosaic Virus, Advanced Materials 11 (3) (1999) 253-256. doi:10.1002/(SICI)1521-4095(199903)11:3¡253::AID-ADMA253;3.0.CO;2-7.

URL http://doi .wiley.com/10.1002/(SICI) 1521-4095(199903) 11:3<253: :AID-ADMA253>3 . 0.C0;2-7

[30] E. Dujardin, C. Peet, G. Stubbs, J. N. Culver, S. Mann, Organization of Metallic Nanoparticles Using Tobacco Mosaic Virus Templates, Nano Letters 3 (3) (2003) 413-417.

[31] M. Knez, M. Sumser, A. M. Bittner, C. Wege, H. Jeske, T. P. Martin, K. Kern, Spatially Selective Nucleation of Metal Clusters on the Tobacco Mosaic Virus, Advanced Functional Materials 14 (2) (2004) 116-124. doi:10.1002/adfm.200304376.

URL http://doi.wiley .com/10.1002/adfm. 200304376

[32] S. Y. Lee, E. Royston, J. N. Culver, M. T. Harris, Improved metal cluster deposition on a genetically engineered tobacco mosaic virus template., Nanotechnology 16 (7) (2005) S435-41. doi:10.1088/0957-4484/16/7/019.

URL http://www.ncbi.nlm.nih.gov/pubmed/21727464

[33] J. S. Lim, S. M. Kim, S. Y. Lee, E. A. Stach, J. N. Culver, M. T. Harris, Quantitative study of Au(III) and Pd(II) ion biosorption on genetically engineered Tobacco mosaic virus., Journal of colloid and interface science 342 (2) (2010) 455-61. doi:10.1016/j.jcis.2009.10.028.

URL http://www.ncbi.nlm.nih.gov/pubmed/19914631 
[34] A. K. Manocchi, N. E. Horelik, B. Lee, H. Yi, Simple, readily controllable palladium nanoparticle formation on surface-assembled viral nanotemplates., Langmuir : the ACS journal of surfaces and colloids 26 (5) (2010) 3670-7. doi:10.1021/la9031514.

URL http://www.ncbi.nlm.nih.gov/pubmed/19919039

[35] K. M. Bromley, A. J. Patil, A. W. Perriman, G. Stubbs, S. Mann, Preparation of high quality nanowires by tobacco mosaic virus templating of gold nanoparticles, Journal of Materials Chemistry 18 (40) (2008) 4796. doi:10.1039/b809585j.

URL http://xlink.rsc.org/?DOI=b809585j

[36] A. S. Freer, L. Mueller, C. Gilpin, M. T. Harris, A novel method to determine the resistance of biotemplated nanowires, Chemical Engineering Communicationsdoi:10.1080/00986445.2014.912637.

[37] C. Yang, S. Jung, H. Yi, A biofabrication approach for controlled synthesis of silver nanoparticles with high catalytic and antibacterial activities, Biochemical Engineering Journal 89 (2014) 10-20. doi:10.1016/j.bej.2013.12.008.

URL http://linkinghub.elsevier.com/retrieve/pii/S1369703X13003446

[38] C. Yang, J. H. Meldon, B. Lee, H. Yi, Investigation on the catalytic reduction kinetics of hexavalent chromium by viral-templated palladium nanocatalysts, Catalysis Today 233 (2014) 108-116. doi:10.1016/j.cattod.2014.02.043.

URL http://linkinghub.elsevier.com/retrieve/pii/S0920586114001977

[39] E. Dujardin, S. Mann, Synthesis and Assembly of Nanoparticles and Nanostructures Using Bio-Derived Templates, 2007.

[40] J. S. Lim, S. M. Kim, S. Y. Lee, E. A. Stach, J. N. Culver, M. T. Harris, Surface functionalized silica as a toolkit for studying aqueous phase palladium adsorption and mineralization on thiol moiety in the absence of external reducing agents., Journal of colloid and interface science 356 (1) (2011) 31-6. doi:10.1016/j.jcis.2010.12.064. URL http://www.ncbi.nlm.nih.gov/pubmed/21272887

[41] J. M. Slocik, R. R. Naik, M. O. Stone, D. W. Wright, Viral templates for gold nanoparticle synthesis, Journal of Materials Chemistry 15 (7) (2005) 749. doi:10.1039/b413074j.

URL http: //xlink.rsc .org/?DOI=b413074j

[42] C. Y. Chiu, Y. Li, Y. Huang, Size-controlled synthesis of Pd nanocrystals using a specific multifunctional peptide., Nanoscale 2 (6) (2010) 927-30. doi:10.1039/c0nr00194e.

URL http://www.ncbi.nlm.nih.gov/pubmed/20648291

[43] K. T. Nam, . Y. J. Lee, . E. M. Krauland, S. T. Kottmann, A. M. Belcher, Peptide-Mediated Reduction of Silver Ions on Engineered Biological Scaffolds, ACS nano 2 (7) (2008) 1480-1487.

[44] J. S. Lim, S. M. Kim, S. Y. Lee, E. A. Stach, J. N. Culver, M. T. Harris, Biotemplated aqueous-phase palladium crystallization in the absence of external reducing agents., Nano letters 10 (10) (2010) 3863-7. 
doi:10.1021/nl101375f.

URL http://www.ncbi.nlm.nih.gov/pubmed/20845914

[45] C. Yang, C. H. Choi, C. S. Lee, H. Yi, A facile synthesis-fabrication strategy for integration of catalytically active viral-palladium nanostructures into polymeric hydrogel microparticles via replica molding, ACS Nano 7 (6) (2013) 5032-5044.

[46] J. Panchompoo, L. Aldous, R. G. Compton, Irreversible uptake of palladium from aqueous systems using 1-cysteine methyl ester physisorbed on carbon black, Journal of Materials Chemistry 21 (26) (2011) 9513. doi:10.1039/c0jm04493h.

URL http://xlink.rsc.org/?DOI=c0jm04493h

[47] S. Ayyappan, Nanoparticles of Ag, Au, Pd, and Cu produced by alcohol, Journal of Materials Research 12 (2) (1997) 398-401.

[48] X. Zhou, L. Zheng, R. Li, B. Li, S. Pillai, P. Xu, Y. Zhang, Biotemplated fabrication of size controlled palladium nanoparticle chains, Journal of Materials Chemistry 22 (18) (2012) 8862. doi:10.1039/c2jm16411f.

URL http: //xlink.rsc . org/?DOI=c2jm16411f

[49] N. Toshima, M. Harada, K. Kushibashi, K. Asakurat, Catalysts by Using Extended X-ray Absorption Fine Structure Spectroscopy (14) (1991) 1448-1453.

[50] C. M. Lin, T. L. Hung, Y. H. Huang, K. T. Wu, M. T. Tang, C. H. Lee, C. Chen, Y. Chen, Size-dependent lattice structure of palladium studied by x-ray absorption spectroscopy, Physical Review B 75 (12) (2007) 125426. doi:10.1103/PhysRevB.75.125426.

URL http://link.aps.org/doi/10.1103/PhysRevB.75.125426

[51] C. H. Chen, L. S. Sarma, J. M. Chen, S. C. Shih, G. R. Wang, D. G. Liu, M. T. Tang, J. F. Lee, B. J. Hwang, Architecture of Pd Au Bimetallic Nanoparticles in Sodium Bis(2-ethylhexyyl)solfosuccinate Reverse Micelles As Investigated by X-ray Absorption Spectroscopy, ACS nano 1 (2).

[52] J. Miller, A. Kropf, Y. Zha, J. Regalbuto, L. Delannoy, C. Louis, E. Bus, J. van Bokhoven, The effect of gold particle size on AuAu bond length and reactivity toward oxygen in supported catalysts, Journal of Catalysis 240 (2) (2006) 222-234. doi:10.1016/j.jcat.2006.04.004.

URL http://linkinghub.elsevier.com/retrieve/pii/S0021951706001205

[53] A. S. Freer, L. Guarnaccio, K. Wafford, J. Smith, J. Steilberg, J. N. Culver, M. T. Harris, SAXS characterization of genetically engineered tobacco mosaic virus nanorods coated with palladium in the absence of external reducing agents., Journal of colloid and interface science 392 (2013) 213-8. doi:10.1016/j.jcis.2012.09.072.

URL http://www.ncbi.nlm.nih.gov/pubmed/23195774

[54] M. A. Watzky, R. G. Finke, Transition Metal Nanocluster Formation Kinetic and Mechanistic Studies . A New Mechanism When Hydrogen Is the Reductant : Slow, Continuous Nucleation and Fast Autocatalytic Surface Growth, Journal of Am. Chem. Society 119 (1997) 10382-10400. 


\section{Supporting Information}

Data in Table 1 shows EXAFS analysis performed with palladium foil and palladium chloride. Spectra were also compared to a palladium oxide reference.

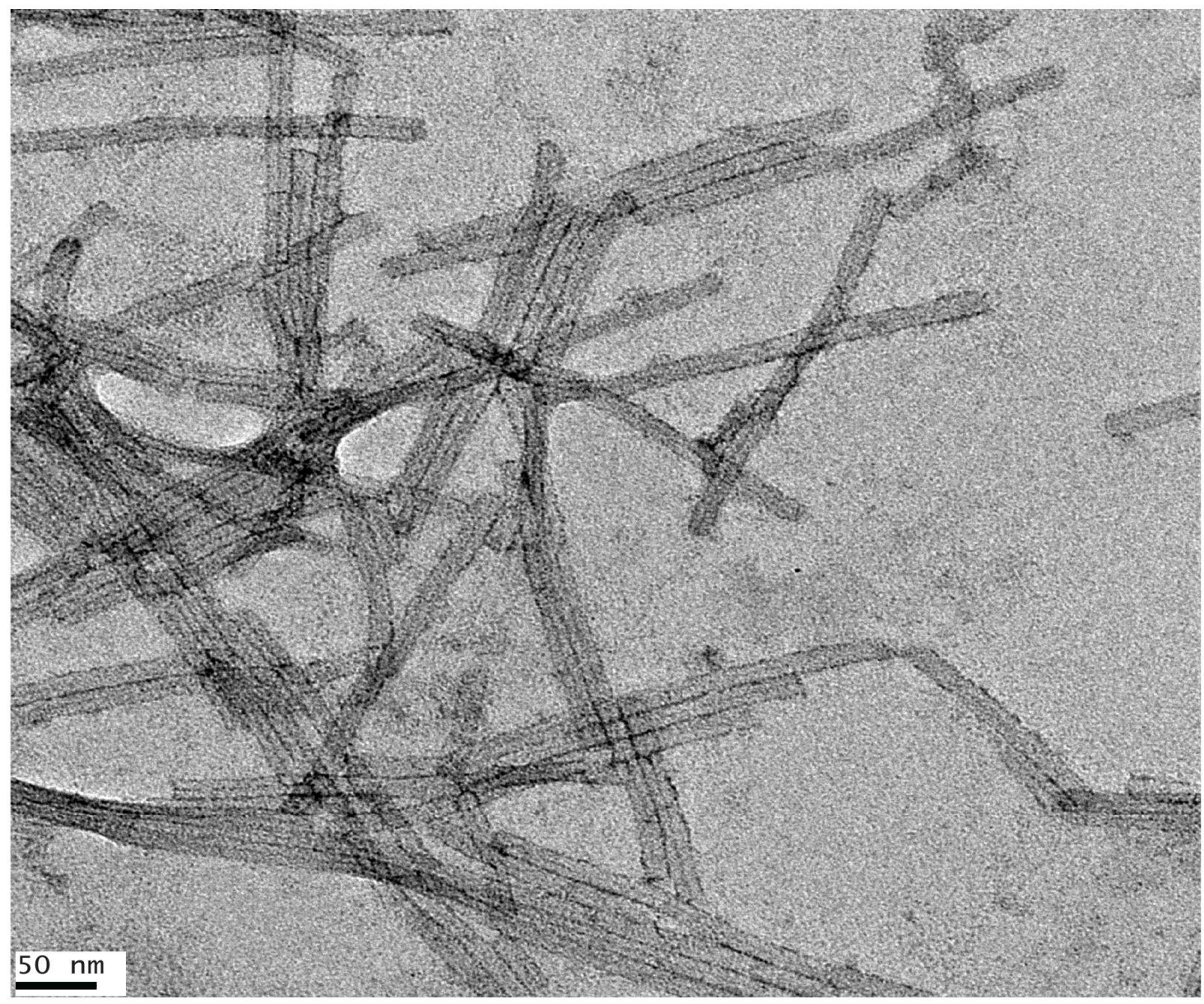

Figure 6. TEM image of $0.4 \mathrm{mg} / \mathrm{ml}$ TMV1Cys stained with Uranyl Acetate 
Table 1. EXAFS Parameters for 24 of 180 times points during experiment.

\begin{tabular}{|c|c|c|c|c|c|c|c|c|}
\hline \multirow{2}{*}{$\begin{array}{l}\text { Time } \\
\text { (hrs) }\end{array}$} & \multicolumn{2}{|c|}{$\mathrm{N}( \pm 10 \%)$} & \multicolumn{2}{|c|}{$\mathrm{R}(\AA)$} & \multicolumn{2}{|c|}{ DWF } & \multicolumn{2}{|c|}{$E_{o}(e V)$} \\
\hline & $(\mathrm{Pd}-\mathrm{Pd})$ & $(\mathrm{Pd}-\mathrm{Cl})$ & (Pd-Pd) & $(\mathrm{Pd}-\mathrm{Cl})$ & (Pd-Pd) & $(\mathrm{Pd}-\mathrm{Cl})$ & $(\mathrm{Pd}-\mathrm{Pd})$ & $(\mathrm{Pd}-\mathrm{Cl})$ \\
\hline 2.0 & - & 4 & - & 2.28 & - & 0.0027 & - & -1.7 \\
\hline 2.1 & 1.0 & 2.27 & 2.75 & 2.28 & 0.001 & 0.0027 & -1.94 & -3.6 \\
\hline 2.5 & 3.0 & 2.65 & 2.75 & 2.28 & 0.001 & 0.0027 & 2.0 & -1.9 \\
\hline 2.6 & 3.6 & 2.0 & 2.75 & 2.28 & 0.001 & 0.0027 & -0.90 & -1.7 \\
\hline 2.8 & 4.9 & 1.9 & 2.75 & 2.28 & 0.001 & 0.0027 & 0.60 & -2.8 \\
\hline 2.9 & 4.8 & 1.6 & 2.75 & 2.28 & 0.001 & 0.0027 & -0.90 & -3.3 \\
\hline 3.1 & 5.8 & 1.7 & 2.75 & 2.28 & 0.001 & 0.0027 & 0.07 & -2.5 \\
\hline 3.3 & 6.1 & 1.4 & 2.75 & 2.28 & 0.001 & 0.0027 & 0.26 & -2.5 \\
\hline 3.4 & 7.1 & 0.8 & 2.75 & 2.28 & 0.001 & 0.0027 & 0.32 & -2.0 \\
\hline 3.6 & 7.4 & 1.2 & 2.75 & 2.28 & 0.001 & 0.0027 & 0.29 & -2.0 \\
\hline 3.8 & 7.4 & 1.45 & 2.75 & 2.28 & 0.001 & 0.0027 & 0.30 & -3.1 \\
\hline 3.9 & 7.5 & 1.5 & 2.75 & 2.28 & 0.001 & 0.0027 & -0.24 & -5.1 \\
\hline 4.1 & 7.1 & 1.6 & 2.75 & 2.28 & 0.001 & 0.0027 & -0.80 & -2.7 \\
\hline 4.7 & 7.7 & 1.2 & 2.75 & 2.28 & 0.001 & 0.0027 & -0.46 & -1.3 \\
\hline 5.4 & 8.3 & 0.8 & 2.75 & 2.28 & 0.001 & 0.0027 & 0.21 & -2.4 \\
\hline 6.0 & 8.5 & 0.8 & 2.75 & 2.28 & 0.001 & 0.0027 & 0.13 & -4.0 \\
\hline 6.7 & 8.7 & - & 2.75 & - & 0.001 & - & -0.95 & - \\
\hline 7.2 & 9.1 & - & 2.75 & - & 0.001 & - & -0.31 & - \\
\hline 7.6 & 9.5 & - & 2.75 & - & 0.001 & - & 0.77 & - \\
\hline 8.6 & 9.4 & - & 2.75 & - & 0.001 & - & -1.0 & - \\
\hline 9.3 & 10.4 & - & 2.75 & - & 0.001 & - & 0.80 & - \\
\hline 10.6 & 10.9 & - & 2.75 & - & 0.001 & - & -1.1 & - \\
\hline 11.9 & 10.5 & - & 2.75 & - & 0.001 & - & -0.43 & - \\
\hline 12.5 & 11.0 & - & 2.75 & - & 0.001 & - & 0.26 & - \\
\hline 13.0 & 11.0 & - & 2.75 & - & 0.001 & - & -0.30 & - \\
\hline
\end{tabular}

$\mathrm{N}=$ Coordination number, ${ }^{3} \mathrm{R}=$ bond length,${ }^{4} \mathrm{DW} \mathrm{G}=$ Debye Waller factor, ${ }^{5} E_{o}(\mathrm{eV})=$ Energy shift ${ }^{6}$ 
*3: Graphical Abstract (for review) Induction period

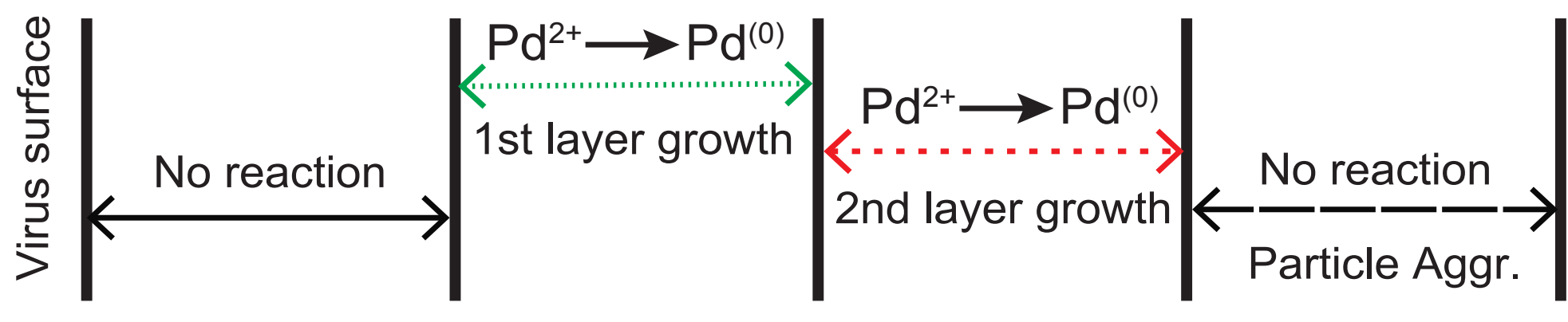

